\title{
OESOPHAGEAL ATRESIA WITH DOUBLE TRACHEO-OESOPHAGEAL FISTULA*
}

\author{
BY
}

\author{
FRITZ REHBEIN \\ From the Surgical Department of the Children's Hospital, Bremen, Germany
}

During the past few years the treatment of oesophageal atresia has made much progress. Published reports deal mainly with the common type which occurs in $85-93 \%$ of all cases. In addition, the difficult problems of atresia without fistula, especially questions of colonic transplantation, have been brought a little nearer a solution (Sherman and Waterston, 1957; Pettersson, 1961; Linder and Hecker, 1962). However, there are still many diagnostic problems, especially those regarding the $\mathrm{H}$-type fistula. The frequency of atresias combined with upper and lower fistula is stated to be between 0.9 and $1.5 \%$ (Fig. 1). Little has been reported on this type, and we have found only about 20 cases among the published papers. The real number is probably very much higher. Most of these children died soon after operation; others, particularly those with only a small fistula, lived for weeks or even months.

\section{Material}

Of 238 cases with oesophageal atresia seen in this unit since 1951 , five $(2 \cdot 1 \%)$ had double tracheooesophageal fistula (Fig. 1). Three of these five children are still alive and completely normal.

\section{Case Reports}

Most cases with double fistula are detected at autopsy, and this was so with our first case in 1955.

Case 1. This child was admitted six days after birth with severe bilateral pneumonia. An attempt at an anastomosis was not made.

Case 2. This child was admitted 17 hours after delivery. During the radiographic examination of the upper pouch the bronchial tree also became outlined although only $2 \mathrm{ml}$. of contrast material was used (Fig. 2). We assume that the material entered the trachea through the upper fistula and not by flowing over. The lower segment was divided near the trachea and the tracheal

* A paper read at a meeting of the British Association of Paediatric Surgeons in Sheffield, July 1963. fistula was closed. It was found that the upper pouch became distended with each passive respiration and collapsed during expiration. When we opened the upper pouch we could smell the ether and we could see the incised edge of the pouch moving with the rhythm of respiration. These findings confirmed the presence of an upper fistula (Fig. 3).

We decided to leave this second fistula alone for the time being for the following reasons. (1) The upper pouch overhung the lower fistula, and we would have had to dissect the rather firm connexions between the pouch and the lower oesophagus to reach the upper fistula. To avoid this, we preferred to leave a small piece of the distal segment. (2) The upper pouch barely reached into the thoracic cavity, so the preparation and closure of the upper fistula from below would have been extremely difficult. For these reasons we made a primary anastomosis and postponed the closure of the upper fistula until a later stage.

Soon after the operation the typical symptoms of an $H$-fistula or the recurrence of a closed fistula began. The abdomen was distended with air and the child frequently aspirated. Tracheostomy improved the condition a little.

Two months after the first operation the upper fistula was closed by right cervical incision. The condition remained satisfactory; the tracheostomy tube was removed and the child was discharged two months later. Several months later severe bouts of coughing occurred together with stridor and cyanosis, and death occurred at the age of 1 year during one of these attacks. Autopsy revealed that death had resulted from choking due to a cyst of the larynx (Fig. 4). This diagnosis had not been made during life. The former junctions of both fistulas with the trachea could be seen. There was no remaining connexion to the oesophagus. Fig. 4 shows that both fistulas led into the trachea above the carina.

Case 3. In this child also, we were able to see the distension of the upper pouch with respiration. The anatomical findings were the same as those in Case 2. The short upper pouch overhung the lower fistula. An anastomosis was made but, in view of our experience with the earlier case, we closed the upper fistula immediately by cervical incision after closing the thoracic cavity. The following day we had to do a tracheostomy and five days later a laparotomy for duodenal stenosis. The child 


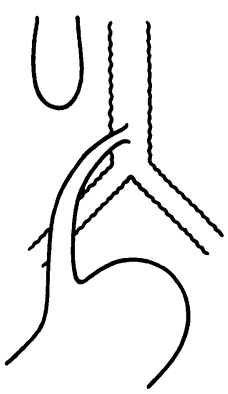

$85 \%-93 \%$

220 cases $=92.4 \%$

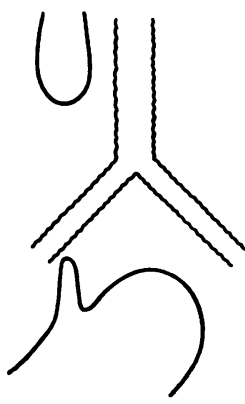

$3 \%-9.8 \%$

8 cases $=3.4 \%$

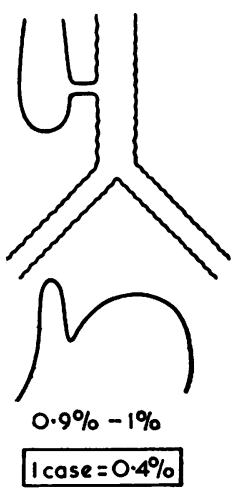

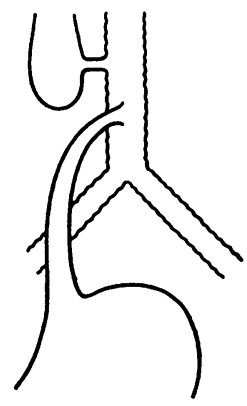

$0.9 \%-1.5 \%$

5 coses $=2.1 \%$

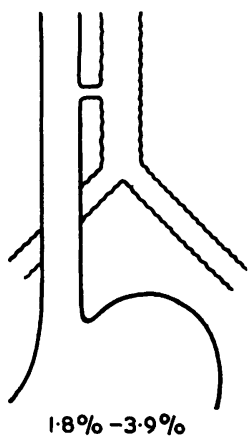

4 coses $=1.7 \%$

FIG. 1.-A survey of the frequency of the different types of oesophageal atresia and $\mathbf{H}$-fistula as reported in the literature and found in our own material.

survived these operations and was discharged after a few months. He is now 3 years old and in a satisfactory condition.

Case 4. An x-ray examination of the upper pouch by contrast material showed simultaneous filling of the distal oesophagus and bronchial tree. During operation we found a similar situation to that described in the preceding cases. The upper fistula was closed by cervical incision, but air entered the right thoracic space. We could not control the pneumothorax and the atelectasis by aspiration and so we reopened the thorax and expanded the lungs. The Engström respirator had to be used for a time during the post-operative period, but the child's further course was without incidence. He is now 18 months old and in good health.

Case 5. In this child also the bronchial tree was outlined on the radiograph. She was operated on as already described in the earlier cases, and the anatomical findings were similar. Her post-operative course was without incidence.

\section{Discussion}

The following observations regarding the diagnosis of an upper fistula are based on these five cases.

If severe bilateral pneumonia occurs soon after birth in a newborn baby who has oesophageal atresia this may indicate the presence of an upper fistula. In addition, if while examining the upper pouch with only a small amount of contrast material, the trachea and bronchial tree are filled, the possibility of an upper fistula exists. However, this latter is not foolproof since the contrast material could be aspirated by overflowing through the larynx.

It is inadvisable to attempt to see the upper fistula, and it is very unlikely that the fistula could be seen even by tracheoscopic examination and injection of methylene blue into the upper pouch. In any case these investigations are not without danger. Roberts (1958) stated that in the presence of an 


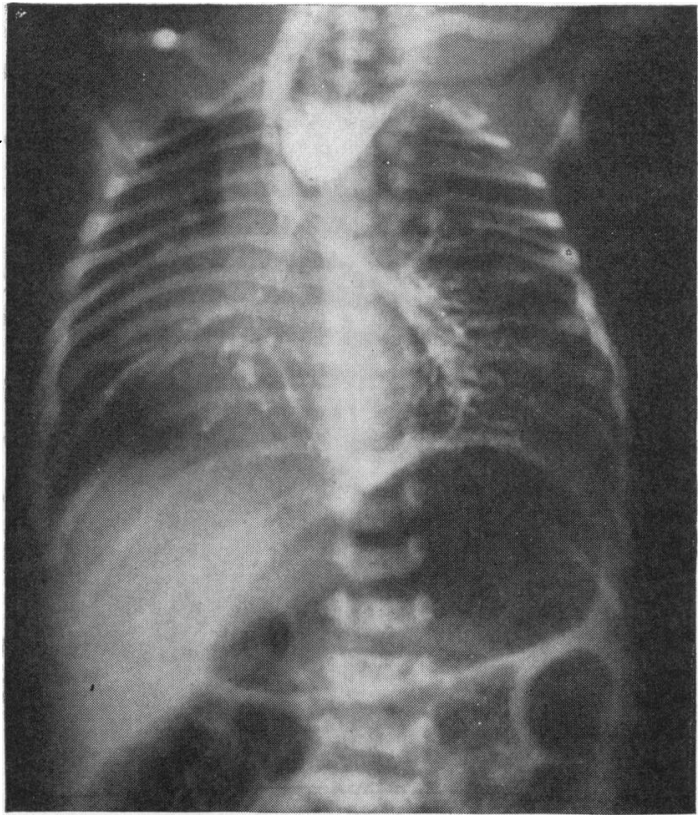

FIG. 2.-Simultaneous filling of the upper pouch and the bronchial tree. Such a finding suggests an upper fistula.

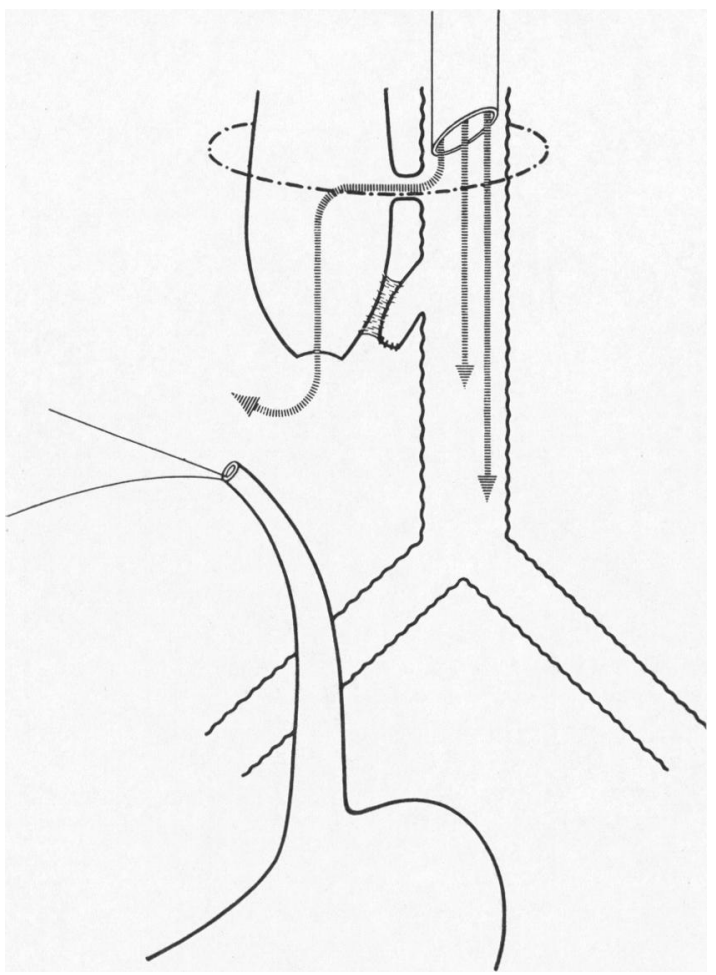

Fig. 3.-Lower fistula closed. Part of the anaesthetic gas escapes out of the upper pouch via the upper fistula.

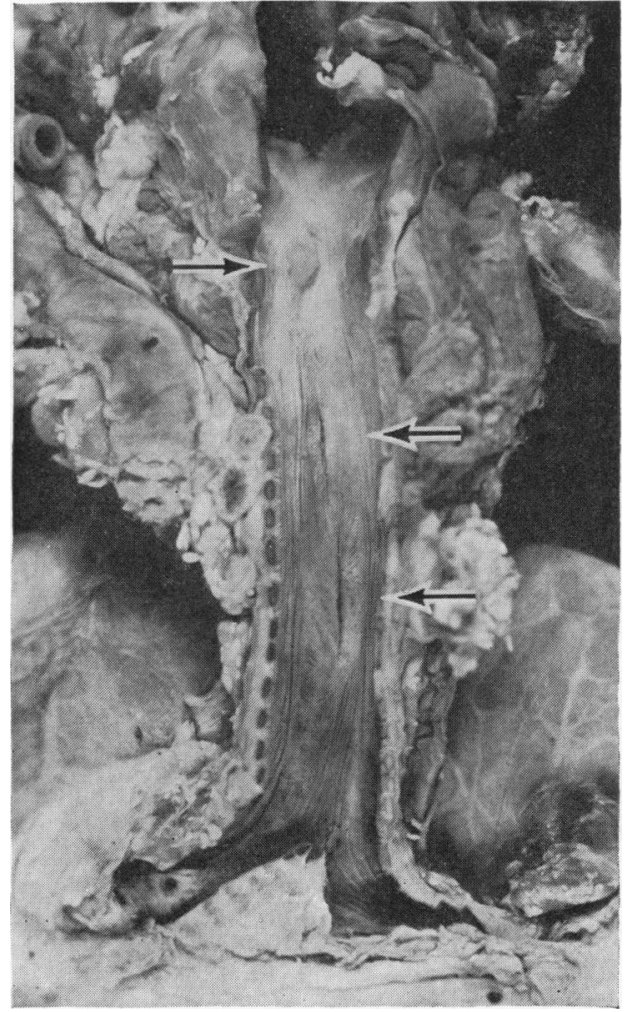

Fig. 4.-Post-mortem findings in the larynx showing the cyst $(\rightarrow)$. Also shown are the openings of the two fistulas high above the carina $(\leftarrow)$.

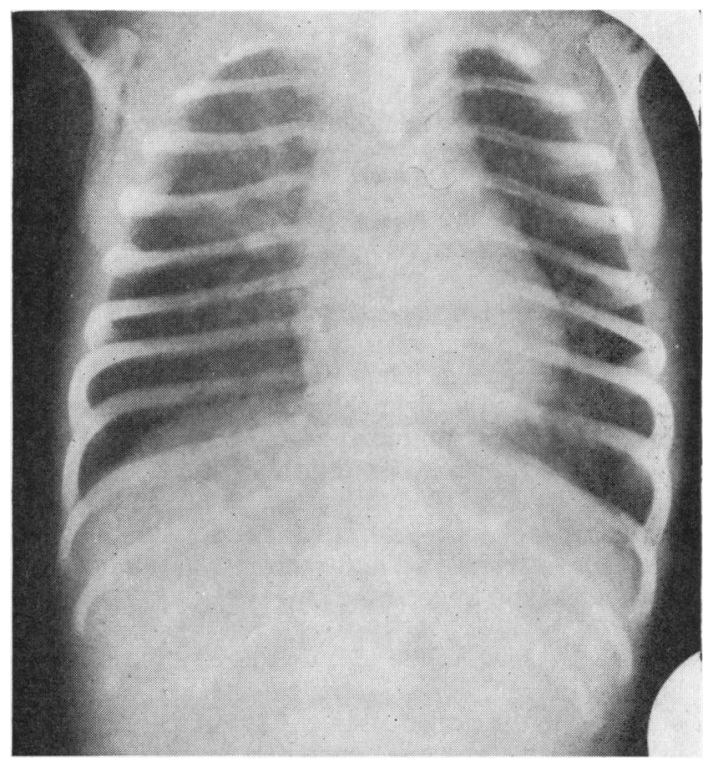

FIG. 5.-Only the small upper pouch may be a sign of the existence of an upper fistula. 


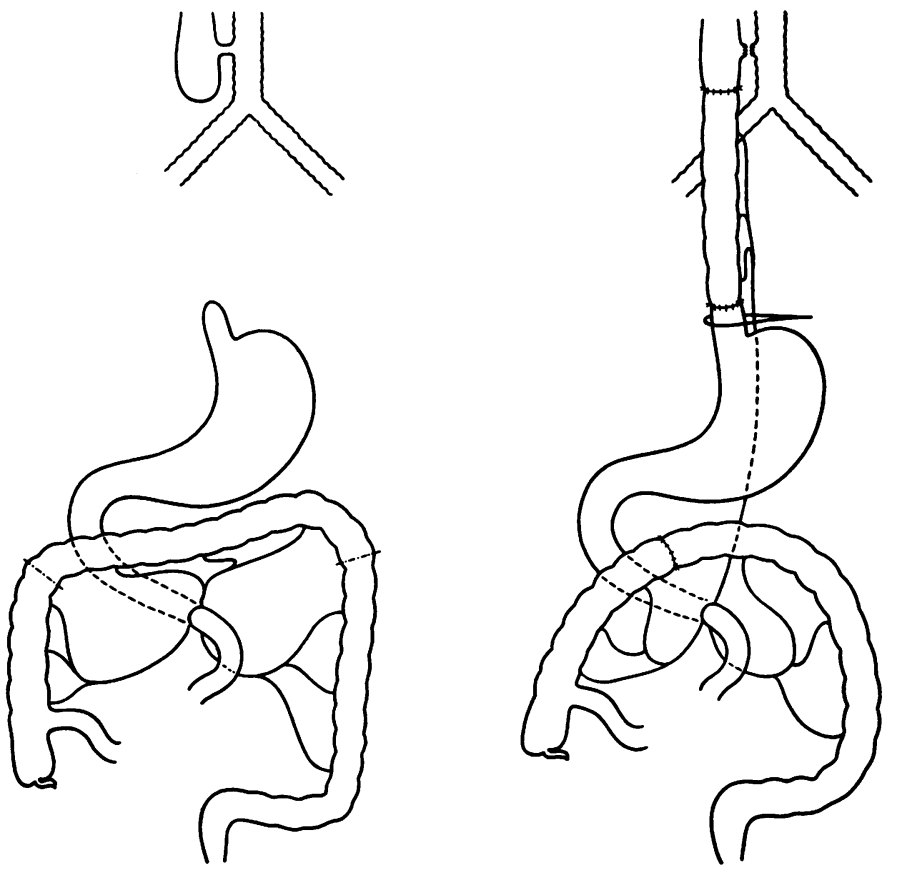

FIG. 6.-Primary reconstruction of the oesophagus by the transverse colon. Upper and lower pouch could be connected with the transplanted transverse colon. Upper fistula closed in a second stage from a cervical approach.

upper fistula the upper pouch was not enlarged, but this was the case in only one of our patients.

The essential part of the diagnosis has to be made during the operation. If the distal segment communicates with the trachea high up, and if it is overlapped by the upper pouch, the possibility of an upper fistula exists. These findings were observed in four of our five cases.

If the upper pouch expands synchronously with breathing and if after opening it the edge of the incision moves in the same rhythm, an upper fistula is likely, although not always certain: we saw it once in a child and consequently did an additional cervical operation, but did not find any upper fistula. On the other hand, this sign might not be seen if the fistula is very small and blocked by mucus or even blocked by the endotracheal tube (Krediet, 1955).

Such signs as early onset of bilateral pneumonia, outlining of the bronchial tree during x-ray examination of the upper pouch, the described anatomical findings and the observations on the upper pouch during respiration, are not absolutely reliable alone. But if all or some of them appear together, there is a strong probability that an upper fistula exists, and I believe that the observation of these points is sufficient for making the right diagnosis. This type of oesophageal atresia is so rare that making more diagnostic efforts routinely before operation does not seem justifiable in order to find an upper fistula. There is no disadvantage to the patient if the final diagnosis is made only at operation.

During the post-operative period the suspicion of an overlooked upper fistula must arise if the typical signs appear immediately after the operation. If it is evident that the upper fistula is high, the cervical operation can be made at any time. If signs of a fistula appear on the fourth or fifth post-operative day, a recurrent fistula is more likely.

Regarding the post-operative course it is better to close both fistulas in one step. It is only in very tiny fistulae, which can barely admit air or mucus, that a delay in closure is of no great importance. The same observation can be made in a very tiny $\mathrm{H}$-fistula or in a very small recurrent fistula. But if the fistula is larger, great difficulties can be expected, and these can only be prevented by early operation. It may be a coincidence that in all our four operated cases the same anatomical findings were present, and that the removal of the upper fistula was possible in each case by cervical incision, or, as I believe, only by cervical incision. This is not always the case, since S. J. Cohen (1963, personal communication), 
Haight (1957, 1961), Stephens, Mustard and Simpson (1956) and Konrad and Rotthoff (1958) reported removal by thoracic approach. In the case described by Krediet (1955) this would have been possible too. On the other hand, Humphreys, Hogg and Ferrer (1956) described two cases in which the upper fistula was located in the cervical area. It is very interesting also that according to Lynn and Davis (1961) nearly one-quarter of all $\mathrm{H}$-fistulas are situated in the cervical area too. Fistulas in this site only can be operated on by cervical incision. But even the fistulas located at the junction of the cervical and thoracic parts of the oesophagus can be reached easily from the neck. Very probably in these cases the cervical approach is simpler and easier. Regarding the technical aspect it has to be noted that there should be only very gentle pulling on the oesophagus during the preparation so as not to endanger the thoracic anastomosis. Furthermore there is a risk that in opening the pleural cavity a dangerous pneumothorax may result.

Finally, I want to mention a case in which only an upper fistula was present. The upper pouch was significantly small (Fig. 5), similar to that reported by Roberts (1958). On the second day of life we performed a primary colonic transplantation using the upper and distal oesophageal segments for anastomosis (Fig. 6). The upper fistula was seen when the upper pouch was opened, but it was not closed until five days later, by cervical incision, because the operation in this particular case would have lasted too long. The condition of the child deteriorated due to frequent aspirations through the upper fistula. Marked improvement occurred only after the second operation. Unfortunately, the child died on the thirteenth day after operation from peritonitis, caused by gastric perforation by the feeding tube. The autopsy showed the perfect condition of the three anastomoses and of the site of closure of the upper fistula.

\section{Summary}

The existence of an additional upper oesophagotracheal fistula in cases of oesophageal atresia may easily be overlooked. But it may give rise to serious danger for the child after operation. The problems of diagnosis are discussed in the light of experience with five cases and one case with an upper fistula only. Treatment recommended is closure of the lower fistula, anastomosis and removal of the upper fistula in the same stage. In all cases closure of the upper fistula was carried out through a cervical approach.

\section{REFERENCES}

Haight, C. (1957). Some observations on esophageal atresias and tracheoesophageal fistulas of congenital origin. J. thorac. Surg., 34, 141.

(196i). The management of congenital esophageal atresia and tracheoesophageal fistula. Surg. Clin. N. Amer., 41, 1281.

Humphreys, G. H., Hogg, B. M. and Ferrer, J. (1956). Congenital atresia of esophagus. J. thorac. Surg., 32, 332 .

Konrad, R. M. and Rotthoff, F. (1958). Kritische Gedanken zum Problem der Ósophagusatresie. Z Zbl. Chir., 83, 1902.

Krediet, H. (1955). A case of congenital atresia of the oesophagus with two oesophago-tracheal fistulas. Arch. Chir. neerl., 7, 265.

Linder, F. and Hecker, W. C. (1962). Oesophagusersatz durch Colon. Chirurg., 33, 18.

Lynn, H. B. and Davis, L. A. (1961). Tracheo-esophageal fistula without atresia of the esophagus. Surg. Clin. N. Amer., 41, 871.

Pettersson, G. (1961). Reconstruction of the oesophagus in infancy and childhood. Acta chir. scand., 122, 60.

Roberts, K. D. (1958). Congenital oesophageal atresia and tracheooesophageal fistula. A review of 36 patients. Thorax, 13, 116.

Sherman, C. D., Jr. and Waterston, D. (1957). Oesophageal reconstruction in children using intrathoracic colon. Arch. Dis. Childh., 32, 11.

Stephens, C. A., Mustard, W. T. and Simpson, J. S. (1956). Congenital atresia of the esophagus with tracheo-esophageal fistula. Surg. Clin. N. Amer., 36, 1465. 